\title{
Complex Zeros of the Jonquière or Polylogarithm Function
}

\author{
By B. Fornberg and K. S. Kölbig
}

Abstract. Complex zero trajectories of the function

$$
F(x, s)=\sum_{k=1}^{\infty} \frac{x^{k}}{k^{s}}
$$

are investigated for real $x$ with $|x|<1$ in the complex s-plane. It becomes apparent that there exist several classes of such trajectories, depending on their behaviour for $|x| \rightarrow 1$. In particular, trajectories are found which tend towards the zeros of the Riemann zeta function $\zeta(s)$ as $x \rightarrow-1$, and approach these zeros closely as $x \rightarrow 1-\rho$ for small but finite $\rho>0$. However, the latter trajectories appear to descend to the point $s=1$ as $\rho \rightarrow 0$. Both, for $x \rightarrow-1$ and $x \rightarrow 1$, there are trajectories which do not tend towards zeros of $\zeta(s)$. The asymptotic behaviour of the trajectories for $|x| \rightarrow 0$ is discussed. A conjecture of Pickard concerning the zeros of $F(x, s)$ is shown to be false.

1. Introduction. Let $s=\sigma+i t$ and $z=x+i y$ be two complex variables. The Jonquière function can then be defined by the infinite sum [1], [2]

$$
F(z, s)=\sum_{k=1}^{\infty} \frac{z^{k}}{k^{s}} \quad(|z|<1)
$$

or by the definite integral

$$
\begin{aligned}
& F(z, s)=\frac{z}{\Gamma(s)} \int_{0}^{\infty} \frac{\xi^{s-1}}{e^{\xi}-z} d \xi \\
& (\sigma>0,-\pi<\arg (1-z)<\pi ; \sigma>1, z=1) .
\end{aligned}
$$

This function is a special case of the Lerch transcendent

$$
\Phi(z, s, \alpha)=\sum_{k=0}^{\infty} \frac{z^{k}}{(\alpha+k)^{s}},
$$

namely $F(z, s)=z \Phi(z, s, 1)$. The integral (2) is also called Bose-Einstein, or FermiDirac integral, according to the values of $z$.

These functions have been investigated by several authors, and theoretical results as well as references can be found in the relevant handbooks [1], [2]. In particular, asymptotic expansions of (2) are given by Dingle [3]. For certain special values of the parameters, these functions and some generalizations appear in several fields of

Received December 7, 1973.

AMS (MOS) subject classifications (1970). Primary 33A70; Secondary $10 \mathrm{H} 05$.

Key words and phrases. Jonquière function, polylogarithms, Lerch's transcendent, FermiDirac integrals, Bose-Einstein integrals, Riemann zeta function, Dirichlet series, complex zeros. 
mathematics and applications, occasionally under different names. For instance, if $s=n$ is a positive integer, one has the so-called polylogarithms ( $n$-logarithms, or Spence functions):

$$
S_{n}(z) \equiv L_{n}(z) \equiv L i_{n}(z)=\sum_{k=1}^{\infty} \frac{z^{k}}{k^{n}}=\frac{(-1)^{n}}{(n-2) !} \int_{0}^{1} \frac{\log ^{n-2} \xi \log (1-z \xi)}{\xi} d \xi .
$$

These functions, in particular for small values of $n$, have been investigated by several authors (for references, see Lewin [4]). They have been generalized in an important way in a little-known paper by Nielsen [5] at the beginning of this century. During the last few years, interest in the polylogarithms and the Nielsen functions $S_{n p}(z)$ has revived in two connections. On the one hand, there is the study of the functional relations which they satisfy, especially in relation to other fields of mathematics like group theory and geometry, e.g., Wechsung [6] and in the book of Maier and Kiesewetter [7] for the polylogarithms. On the other hand, there is the importance of these functions in the theory of Feynman integrals in quantum electrodynamics, since they allow closed expressions to be obtained for certain $n$-dimensional integrals over rational functions.

These integrations are very involved but can be handled to a large extent by algebraic manipulation on a computer (see, for example, Maison and Petermann [8]). Problems relating the integration of rational functions and the polylogarithms are also treated by Wechsung [9]. A method for the numerical evaluation of a few Nielsen functions (and polylogarithms) for real $x$ using Chebyshev expansions can be found in [10], and a method for computing $S_{2}(z)$ and $S_{3}(z)$ for complex argument with the help of continued fraction expansions is given by Jacobs and Lambert [11]. Recently, Barlow [12] developed a method for computing $S_{n p}(z)$ for arbitrary complex $z$ using continued fraction approximants.

Our interest in the function $F(z, s)$ lies in another direction. Since, for $z=1$, $F(z, s)$ is identical with the Riemann zeta function

$$
F(1, s)=\zeta(s)=\sum_{k=1}^{\infty} \frac{1}{k^{s}} \quad(\sigma>1)
$$

it would be interesting to know how the zeros of $F(z, s)$, if there are any, approach the zeros of $\zeta(s)$ on the line $\sigma=1 / 2$ as $z \rightarrow 1$, if in fact they do. The problem of the zeros of $F(z, s)$ has been raised by Pickard [13], who, a few years ago, investigated the function $F(z, s)$ for complex $z$ and $s$, noting that but little information about these zeros is available. He stated that "as $z \rightarrow 0$, the zeros are given by

$$
s=\frac{1}{\log 2}[\log |z|+i \arg z+(2 p-1) \pi i] ”
$$

and was "tempted to conjecture that all zeros in the complex $(z, s)$-plane lie on trajectories that lead to the line

$$
\sigma=(\log |z|) /(\log 2)
$$

as $z \rightarrow 0$ and the line $\sigma=1 / 2$ as $z \rightarrow 1$ ". 
It is the aim of this paper to show by theoretical considerations and numerical calculation that both the statement (6) and the conjecture (7) are erroneous. In fact, Pickard has revealed only a very small corner of the amazing and fascinating picture constituted by the zeros of $F(z, s)$.

In order to make the approach to the problem simpler, we restrict ourselves to real values of $z$, namely $-1<x<1, y=0$, and leave the general case for possible further research. We therefore search for trajectories of $F(x, s)=0$ in the complex s-plane, where $x$ is a parameter along these trajectories. Further, it is sufficient to consider only the upper half plane $t>0$.

2. Asymptotes for the Zeros of $F(x, s)$. In order to investigate the behaviour of the zeros of $F(x, s)$ for $x \rightarrow+0$, we rewrite Eq. (1) as

$$
\begin{aligned}
G_{N}(x, s)= & \frac{N^{s}}{x^{N}} F(x, s)=\frac{(N / 1)^{s}}{x^{N-1}}+\frac{(N / 2)^{s}}{x^{N-2}}+\cdots+1+\frac{x}{[(N+1) / N]^{s}} \\
& +\frac{x^{2}}{[(N+2) / N]^{s}}+\cdots=H_{N}(x, s)+R_{N}(x, s),
\end{aligned}
$$

where

$$
\begin{array}{r}
H_{N}(x, s)=1+\frac{x}{[(N+1) / N]^{s}} \\
=1-\exp \left\{-\log (1+1 / N)\left[s-\frac{1}{\log (1+1 / N)}[\log x+(2 P+1) \pi i]\right]\right\} \\
(P=0, \pm 1, \pm 2, \cdots) .
\end{array}
$$

$G_{N}(x, s)$ has the same zeros as $F(x, s)$, apart from $x=0$. It is easily seen that the zeros of $H_{N}(x, s)$ are given for $t>0$ by

$$
\begin{aligned}
\hat{s}_{0}^{+}(x, P, N) & =\frac{1}{\log (1+1 / N)}[\log x+(2 P+1) \pi i] \\
& =u^{+}(x, N)+i v^{+}(P, N)(N=1,2,3, \cdots ; P=0,1,2, \cdots) .
\end{aligned}
$$

We note here that the formula (6) of Pickard corresponds to $N=1$ and is, therefore, a special case of this formula.

Our aim is now to show that, if $x \rightarrow+0$, the quantity $\hat{s}_{0}^{+}(x, P, N)$ is an asymptotic solution of $F(x, s)=0$. For this purpose, we note first that the absolute value of term $k$ in $G_{N}(x, s)$ for $s=\hat{s}_{0}^{+}(x, P, N)$ is given by

$$
\mid \text { term } k \mid=x^{k-N}(N / k)^{u^{+}(x, N)}=x^{f(k, N)},
$$

where

$$
f(k, N)=k-N-\frac{\log (k / N)}{\log (1+1 / N)}
$$


For fixed $N$, and as a function of $k$, it is easy to show that $f(k, N)$ has the following properties:

$$
f(N, N)=f(N+1, N)=0, \quad f(N-1, N)>f(N+2, N)
$$

$$
\left(d^{2} / d k^{2}\right) f(k, N)>0 .
$$

Therefore, it follows that

$$
f(k, N) \geqslant \epsilon_{N}>0 \quad(k \neq N, k \neq N+1)
$$

with

$$
\epsilon_{N}=f(N+2, N)=2-\frac{\log (1+2 / N)}{\log (1+1 / N)} .
$$

In addition, $f(k, N)=O(k)$ for $k \rightarrow \infty$. This leads to the conclusion that

$$
\left|R_{N}(x, s)\right|=O\left(x^{\epsilon}\right)
$$

for $s=\hat{s}_{0}^{+}(x, P, N)$ and $x \rightarrow+0$, independent of $\operatorname{Im} \hat{s}_{0}^{+}=v^{+}(P, N)$. In order to bound $\left|R_{N}(x, s)\right|$ from above uniformly in a strip around the line $\sigma=u^{+}(x, N)$ extending to, say, unit distance on either side of the line, we can proceed as follows. Since |term $k \mid=x^{k-N}(N / k)^{\sigma}$, a change of up to \pm 1 in the value of $\sigma$ can multiply the magnitude of the term by at most $N / k$ or $k / N$, whichever is the larger. This does not affect the uniform convergence $O\left(x^{\epsilon}\right)$ as $x \rightarrow 0$ of the sum $\left|R_{N}(x, s)\right|$ in the infinite strip. We now make use of the following lemmas:

Lemma 1. Given any $\epsilon, 0<\epsilon<2 \pi$, there exists an $\eta>0$ such that $\left|e^{s}-1\right| \geqslant \eta$ for all complex $s$ on the circle $|s|=\epsilon$. In particular, we can choose $\eta$ such that $\lim _{\epsilon \rightarrow 0}(\eta / \epsilon)=1$.

LEMMA 2 (ROUCHÉ). If $f$ and $g$ are regular analytic functions in a simply connected and bounded region $G$, and if $f \neq 0$ and $|f|>|g|$ on a closed path $\Gamma$ completely inside $G$, then $f$ and $f+g$ have the same number of zeros inside $\Gamma$.

We choose a small $\epsilon>0$, where $\epsilon<\log (1+1 / N)$. According to Lemma 1, there exists an $\eta$ such that $\left|H_{N}(x, s)\right| \geqslant \eta$ if $s$ satisfies

$$
\log (1+1 / N)\left|s-\frac{1}{\log (1+1 / N)}[\log x+(2 P+1) \pi i]\right|=\epsilon .
$$

If $x$ now is chosen so small that $\left|R_{N}(x, s)\right|<\eta$ in the strip, then, according to Lemma $2, G_{N}(x, s)=H_{N}(x, s)+R_{N}(x, s)$, and thus $F(x, s)$ has one zero at most at the distance $\epsilon / \log (1+1 / N)$ from $\hat{s}_{0}^{+}(x, P, N)$. Since we could choose $\epsilon$ arbitrarily small, this proves the existence of asymptotes for the zeros when $x \rightarrow+0$. To each $N=1,2$, $3, \cdots$ and each $P=0,1,2, \cdots$ there corresponds one zero given by Eq. (10).

Using $\eta=\epsilon+O(1)$, we find that the distance of the zero of $F(x, s)$ to $\hat{s}_{0}^{+}(x, P, N)$ is of the type const $\cdot x^{\epsilon} N$, where the constant depends on $N$ but not on $x$.

From Eq. (10), we see that the lines

$$
t=v^{+}(P, N)=(2 P+1) \pi / \log (1+1 / N),
$$


which are parallel to the real axis $t=0$, are asymptotes for the zeros $s_{0}^{+}(x, P, N)$ of $F(x, s)$ in the case $x \rightarrow+0$. By considerations similar to those given above, one can find that, in the case $x \rightarrow-0$, the zeros of the corresponding function

$$
H_{N}(x, s)=1-|x| /[(N+1) / N]^{s}
$$

in the upper half plane $t>0$ are given by the expression

$$
\hat{s}_{0}^{-}(x, P, N)=\frac{1}{\log (1+1 / N)}(\log |x|+2 P \pi i)
$$

$$
(N=1,2,3, \cdots ; P=1,2,3, \cdots)
$$

and that the asymptotes of the zeros $s_{0}^{-}(x, P, N)$ of $F(x, s)$ for $-1<x<-0$ are characterized by

$$
t=v^{-}(P, N)=2 P \pi / \log (1+1 / N)
$$

3. Numerical Computation of the Zero Trajectories of $F(x, s)$. The numerical computation of the zeros of $F(x, s)$ was made in double-precision mode (about 28 digits) on a CDC 7600 computer. It was found that straightforward use of the defining sum (1) was convenient for most of the cases. The summation was carried until the modulus of a term became smaller than $10^{-30}$. We started the computation at a parameter value $x=x^{\prime}$ for given values of $P$ and $N$ and took the value $\hat{s}_{0}\left(x^{\prime}, P, N\right)$ as an approximation to the zero $s_{0}\left(x^{\prime}, P, N\right)$ of $F(x, s)$. This value was then refined with a Newton method [14] to at least five decimals, and the value thus obtained was taken as an approximation for $s_{0}\left(x^{\prime}+\Delta x, P, N\right)$, and so on. It was found that the choice

$$
x^{\prime}=10^{-N-2} ; \Delta x=\min \left(10^{[\lg x-x-1]}, 0.01\right),
$$

where $[\xi]$ is the integer part of $\xi$, led in most cases to an efficient and stable computation along the trajectories giving, in addition, convenient values for $x$. In regions where the behaviour of the trajectories was found to be complicated, much smaller values of $\Delta x$ were used. For $N \geqslant 9$, it became more and more difficult to start with $x^{\prime}=$ $10^{-N-2}$, and the trajectories were calculated backwards starting near a zero of $\zeta(s)$. With these methods, the trajectories were computed without great difficulties up to $|x|=0.96$. In the imaginary direction of the $s$-plane, we restricted the calculation arbitrarily to $0<t \leqslant 110$.

4. The Results. We first described the results for the case $+0<x<1$. Here we found two distinct classes of trajectories, characterized by their behaviour for $x \rightarrow 1$. One class does not approach the zeros of $\zeta(s)$ on the line $\sigma=1 / 2$, but tends directly towards the point $s=1$. We computed seven of these trajectories and found that they belong to asymptotes having $P=0, N=1,2, \cdots, 7$. They are shown in Fig. 1, giving a very regular picture. Although it is advisable to be very careful in formulating conjectures in the matter treated here, we believe that this class is characterized by $P=0$, 


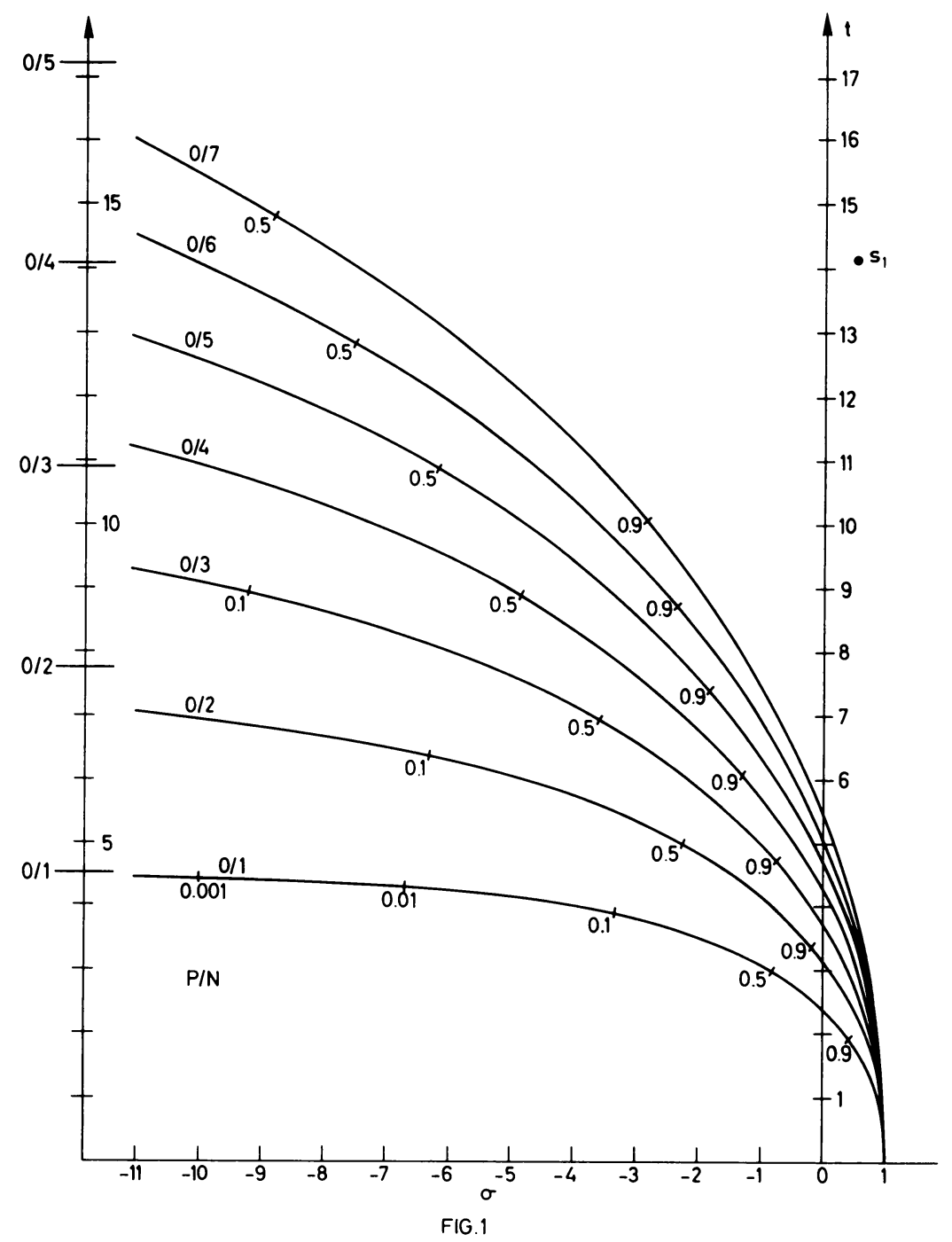

and that all the curves $s_{0}^{+}(x, 0, N)$ will be found to belong to it. It is, however, probable that the regular picture will be destroyed when these trajectories pass, for large $N$, near a zero of $\zeta(s)$.

The other class is characterized by the fact that all the computed trajectories very closely approach a zero of $\zeta(s)$ on the line $\sigma=1 / 2$ as $x \rightarrow 1-\rho$ for small values of $\rho>0$. These curves are shown in Fig. 2, plotted as full (unbroken) lines. (The behaviour of the trajectories for $\rho \rightarrow 0$ is discussed in Section 5.) The zeros $s_{m}$ of $\zeta(s)$ are taken from Haselgrove and Miller [15]. The picture presented by this class is complicated and becomes more and more involved for increasing imaginary part $t$. Nevertheless, some of the curiosities are especially worth mentioning. One notices immediately the tongs-like shape of those trajectories which approach zeros of $\zeta(s)$ which are not very far from each other (for example, $s_{4}$ and $s_{5}, s_{7}$ and $s_{8}, s_{9}$ and $s_{10}, s_{13}$ and $s_{14}$, 


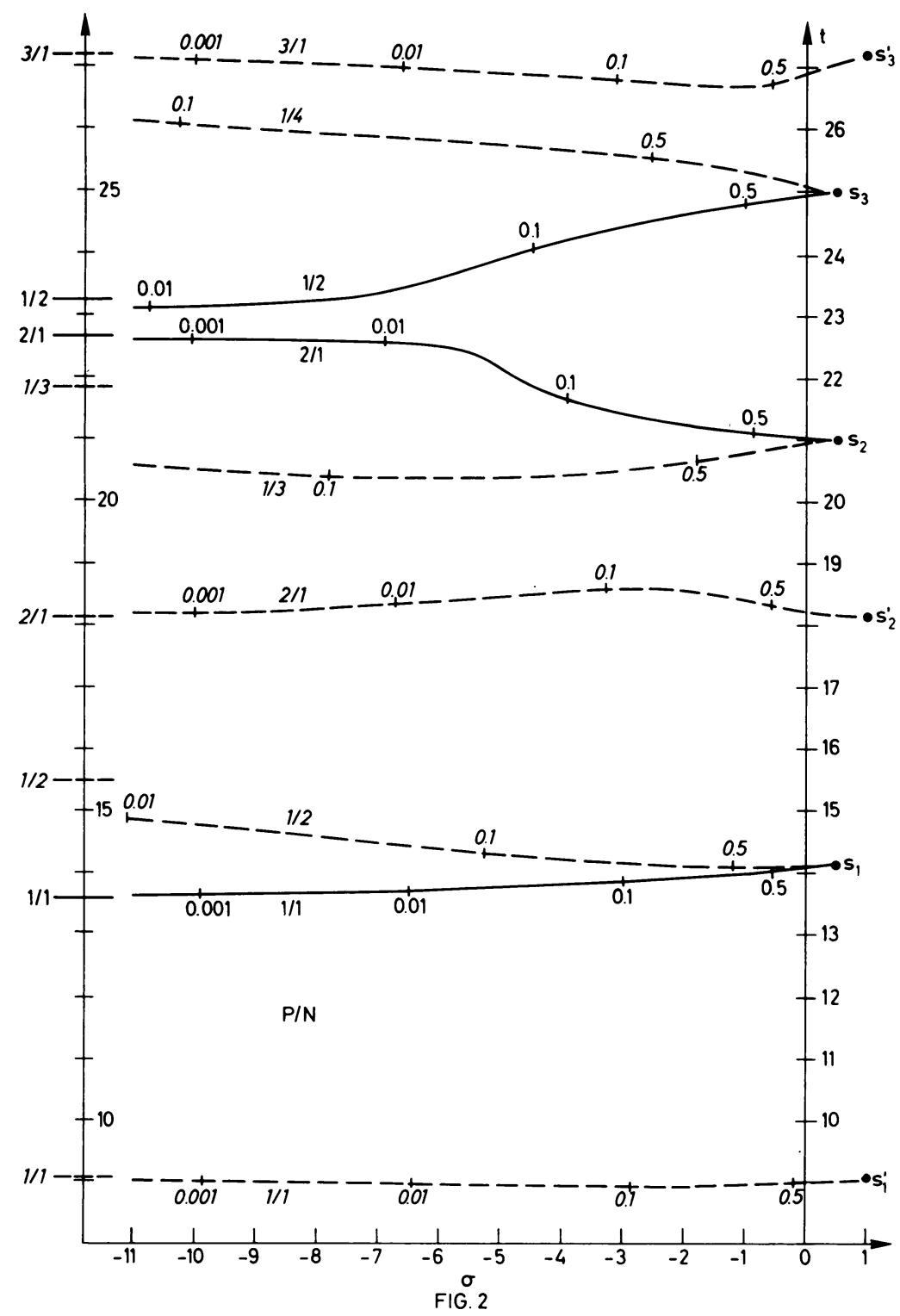

etc.). Further, one finds, at least in the lower part of the region considered, a curious "paired" behaviour of trajectories having $P$ and $N$ interchanged, e.g. the pairs $s_{0}^{+}(x, 1,3)$ and $s_{0}^{+}(x, 3,1), s_{0}^{+}(x, 1,7)$ and $s_{0}^{+}(x, 7,1)$, etc., in particular, $s_{0}^{+}(x, 2,5)$ and $s_{0}^{+}(x, 5,2), s_{0}^{+}(x, 1,9)$ and $s_{0}^{+}(x, 9,1)$, but also, in a different way, $s_{0}^{+}(x, 2,3)$ and $s_{0}^{+}(x, 3,2)$. The asymptotes of these curves are often quite distant from each other, but eventually (outside the picture) these curves separate and approach their asymptotes for $x \rightarrow+0$. Figure 3 shows such behaviour for $s_{0}^{+}(x, 1,8)$ and $s_{0}^{+}(x, 8,1)$. On the other hand, trajectories with $P=N$ are isolated, like $s_{0}^{+}(x, 1,1), s_{0}^{+}(x, 2,2), s_{0}^{+}(x, 3,3)$. Since there can be no interchange of $P$ and $N$ when $P=0$, the exceptional behaviour of the trajectories $s_{0}^{+}(x, 0, N)$, as described above, seems more plausible. 


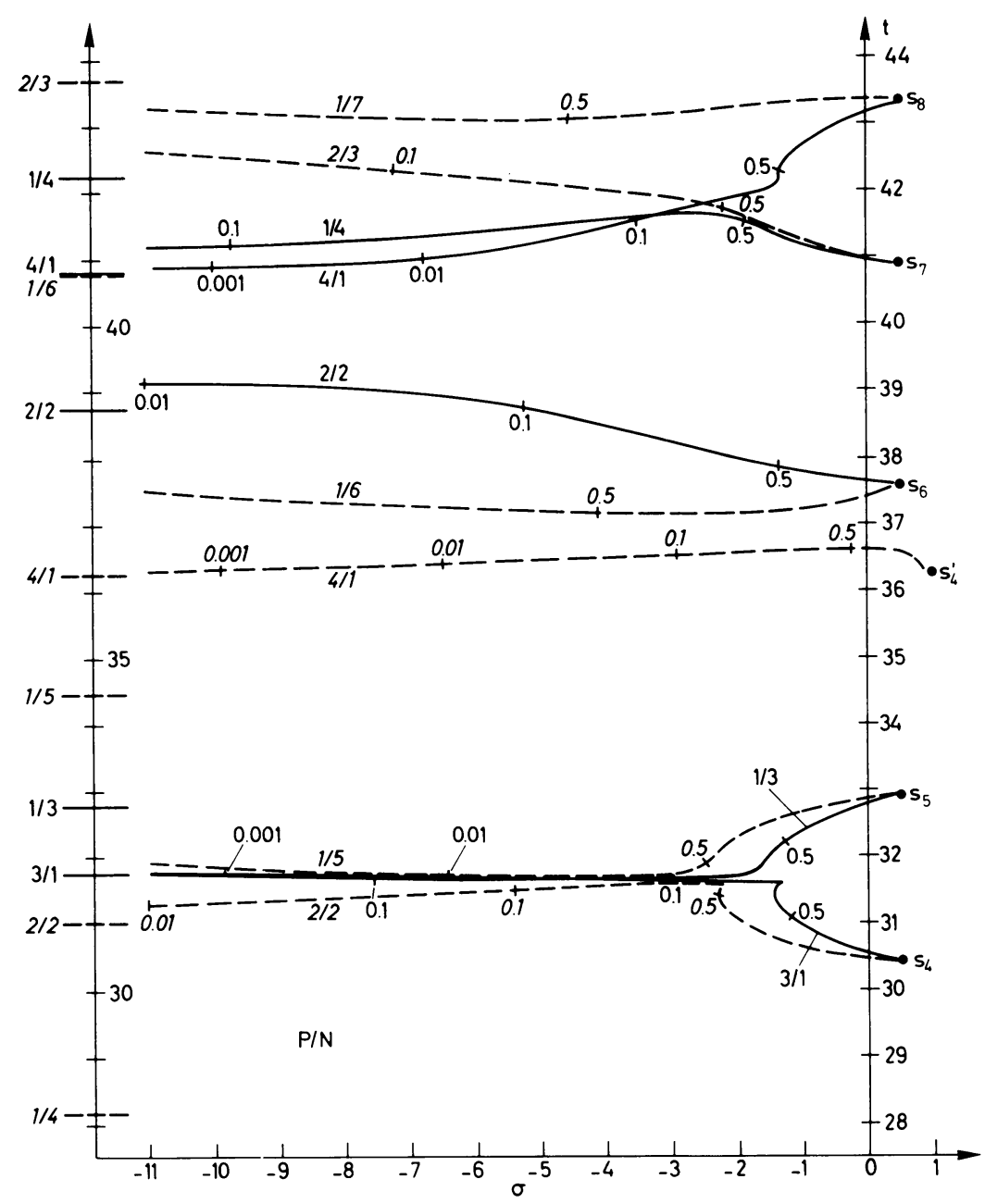

FIG. 2 (cont.)

For large $P$ and $N$, we see from (17) that

$$
v^{+}(P, N) \sim 1 / 2(2 N+1)(2 P+1) \pi \sim v^{+}(N, P),
$$

which means that two asymptotes, with $P$ and $N$ interchanged, become quite close to each other. However, this does not necessarily mean that the corresponding trajectories have a similar behaviour as they advance through the s-plane or that they approach neighboured zeros $s_{m}$. For instance, $v^{+}(3,4)=98.55$ and $v^{+}(4,3)=98.28$ differ by 0.27 only, whereas the zeros $s_{26}=92.49$ and $s_{31}=103.73$, which are approached by $s_{0}^{+}(x, 4,3)$ and $s_{0}^{+}(x, 3,4)$, respectively, have a much larger distance from each other.

Looking at the trajectories which pass near zeros of $\zeta(s)$ which are not far from each other, one notices a certain "indecisive" behaviour of some of these curves, as if the zeros. of $\zeta(s)$ are trying to "attract" the trajectories. This can lead to very sharp bends, as for instance for $s_{0}^{+}(x, 3,1)$. Figure 4 shows a close-up of this curve near the bend. 


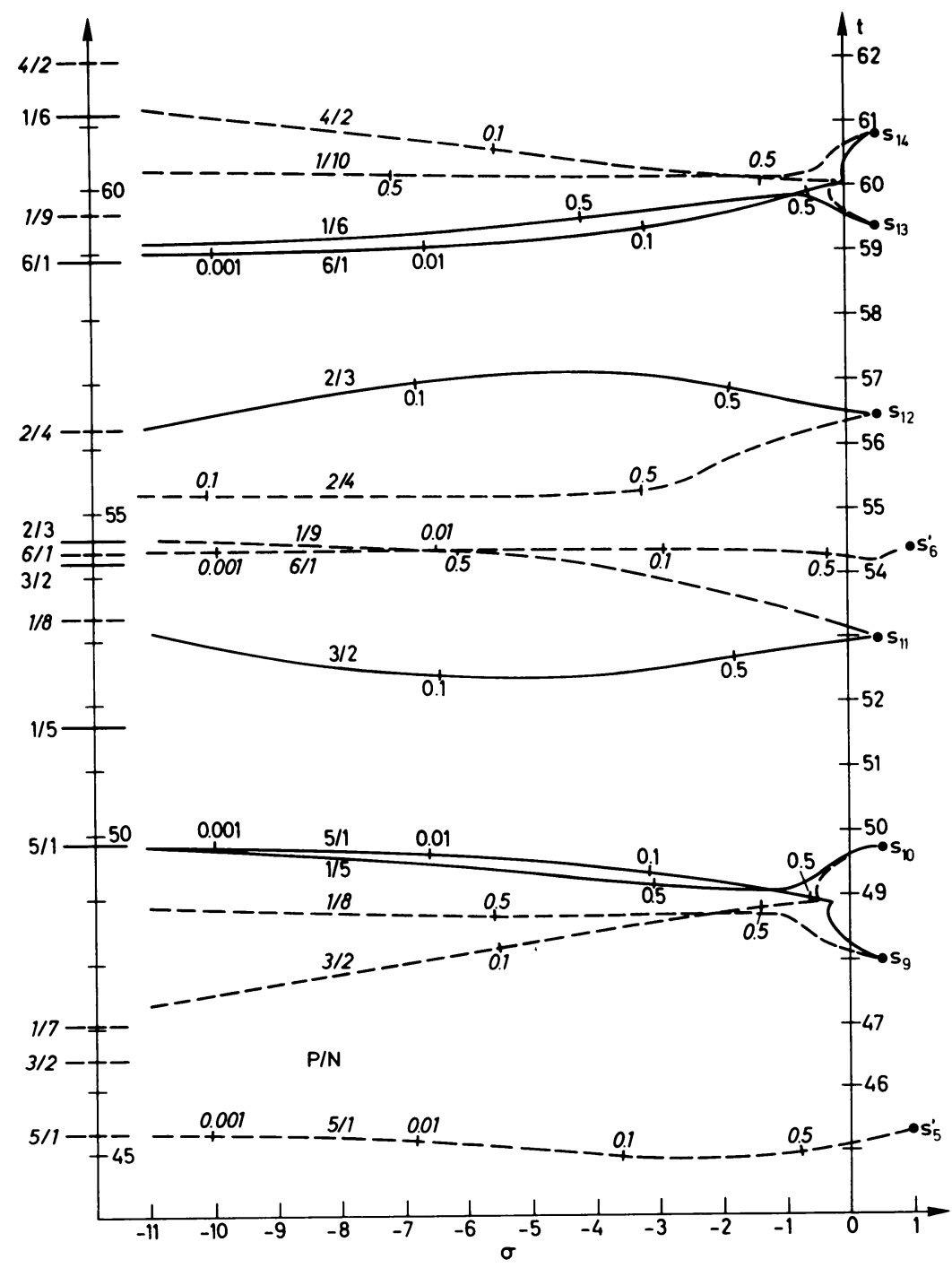

FIG. 2 (cont.)

It is obvious that the behaviour of the trajectories becomes more and more complicated the higher one goes in the s-plane. In particular, there seems to be no way of predicting from $P$ and $N$ the ordinal number $m$ of the zero $s_{m}$ of $\zeta(s)$ which is approached by the trajectory $s_{0}^{+}(x, P, N)$, even if there is a one-to-one correspondence between zeros $s_{m}$ and trajectories coming from asymptotes $v^{+}(P, N)$ which is, of course, an open question. Up to $s_{33}$, however, all zeros of $\zeta(s)$ are approached by exactly one trajectory $s_{0}^{+}(x, P, N)$, and we computed all trajectories belonging to the asymptotes $v^{+}(P, N) \leqslant 110$.

For the case $-0>x>-1$, the trajectories are the broken curves in Fig. 2 . Since

$$
\left(1-2^{1-s}\right) \zeta(s)=\sum_{k=1}^{\infty} \frac{(-1)^{k-1}}{k^{s}} \quad(\sigma>0)
$$




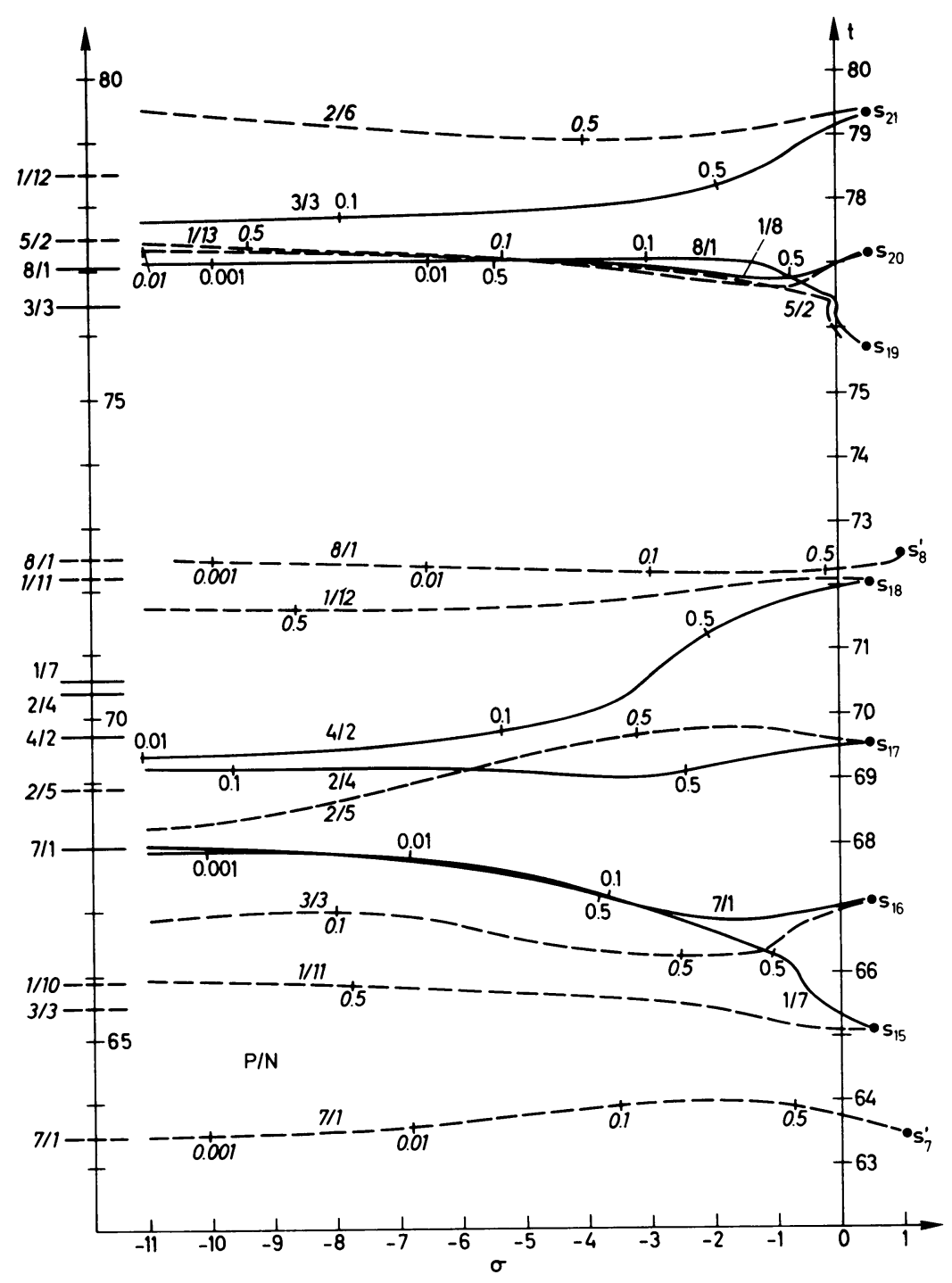

FIG. 2 (cont)

one might expect that, as $x \rightarrow-1$, some of these trajectories would terminate at the equidistant points

$$
s_{m}^{\prime}=1+\frac{2 \pi m}{\log 2} i \approx 1+9.06472 m i \quad(m=1,2, \cdots),
$$

which are the zeros of $\phi(s)=1-2^{1-s}$. This is, indeed, the case. On the other hand, there are also trajectories which approach the zeros of $\zeta(s)$. Therefore, here again we have two classes of trajectories.

It is remarkable that, of all the computed trajectories $s_{0}^{-}(x, P, N)$, only those with $N=1$ tend to a zero $s_{m}^{\prime}$. In fact, the trajectories $s_{0}^{-}(x, P, 1), P=1,2, \cdots, 12$, approach the corresponding $s_{p}^{\prime}$. In addition, we see that

$$
\operatorname{Im} s_{P}^{\prime}=v^{-}(P, 1)=2 P \pi /(\log 2)
$$




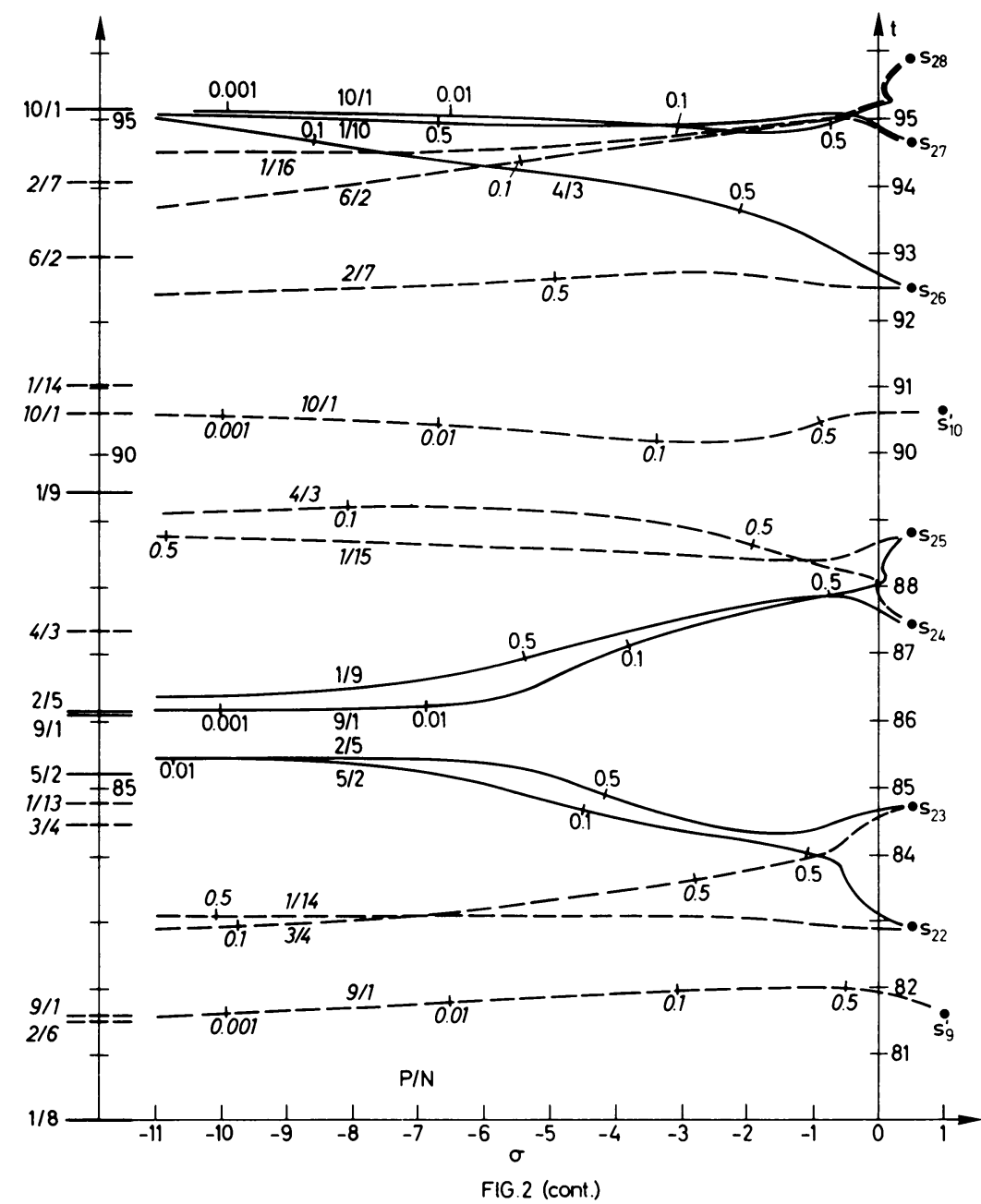

which means that the asymptotes $v^{-}(P, 1)$ have, for all $P$, the same ordinate $t$ as the zeros of $\phi(s)$. Therefore, again with the necessary caution, we conjecture that all trajectories $s_{0}^{-}(x, P, 1)$ will approach the corresponding zeros $s_{P}^{\prime}$. The remaining trajectories, corresponding to $-1<x<-0$, approach zeros of $\zeta(s)$, often in a smoother way than in the case $+0<x<1$. For $P$ and $N$ interchanged, there is no behaviour similar to the $s_{0}^{+}(x, P, N)$, although "pairs" and "tongs" are also present. A very interesting case is $s_{0}^{-}(x, 2,8)$, which, after having directed itself towards $s_{33}$, bends with a sharp loop to $s_{32}$. A close-up view is shown in Fig. 5. As for the case $+0<x<1$, all the zeros of $\zeta(s)$ up to $s_{33}$ are approached by exactly one trajectory $s_{0}^{-}(x, P, N)$. One should note that the trajectory $s_{0}^{-}(x, 1,18)$ has its asymptote $v^{-}(1,18)=116.21$ outside the figure and that the trajectory $s_{0}^{-}(x, 7,2)$, coming from $v^{-}(7,2)=108.47$, approaches an $s_{m}$ with $t>110$ outside the figure.

In order to test our calculations, we applied the well-known theorem

$$
Z(x)=\frac{1}{2 \pi i} \int_{C} \frac{F^{\prime}(x, s)}{F(x, s)} d s
$$




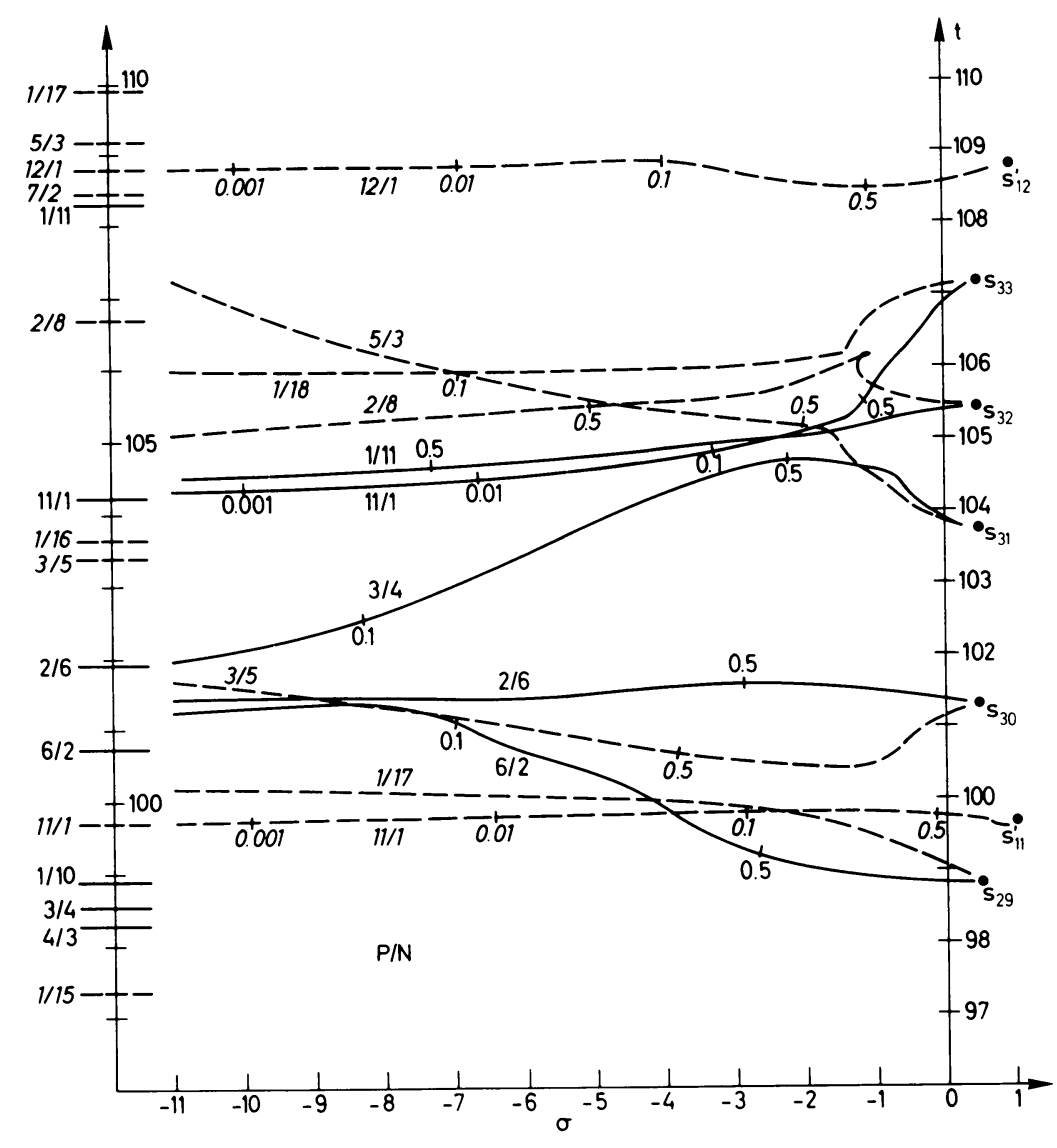

FIG. 2 (cont.)

giving the number $Z(x)$ of zeros of $F(x, s)$ inside a closed contour $C$. The integral was evaluated by numerical integration with an adaptive Gaussian quadrature procedure [16]. We found $Z(0.1)=27$ in the rectangle $(0,110 i,-11+110 i,-11)$ and $Z(-0.1)=26$ in the rectangle $(i, 109 i,-11+109 i,-11+i)$. These values agree with the numbers of computed zeros found in these rectangles (see Fig. 2).

5. Trajectories for $x$ Very Close to One. In this section, we present some heuristic arguments concerning the behaviour of the trajectories $s_{0}^{+}(x, P, N)$ as $x \rightarrow 1$.

We shall use the formula [1]

$$
F(x, s)=\Gamma(1-s)(-\log x)^{s-1}+\zeta(s)+\sum_{n=1}^{\infty} \zeta(s-n) \frac{\log ^{n} x}{n !}
$$

to study the behaviour of the zeros of $F(x, s)$ as $x \rightarrow 1$, especially those which, for $x \rightarrow 1-\rho$, initially approach the nontrivial zeros of $\zeta(s)$. As an example, we take the behaviour of $F(x, s)$ near $s_{1}$. When $x \rightarrow 1$, the sum in (26) becomes negligible as compared to $\zeta(s)$, and the zeros of $F(x, s)$ therefore arise effectively from the cancellation between $\zeta(s)$ and the term

$$
\Psi(x, s)=\Gamma(1-s)(-\log x)^{s-1}=\Gamma(1-s) \exp [(s-1) \log (-\log x)] .
$$



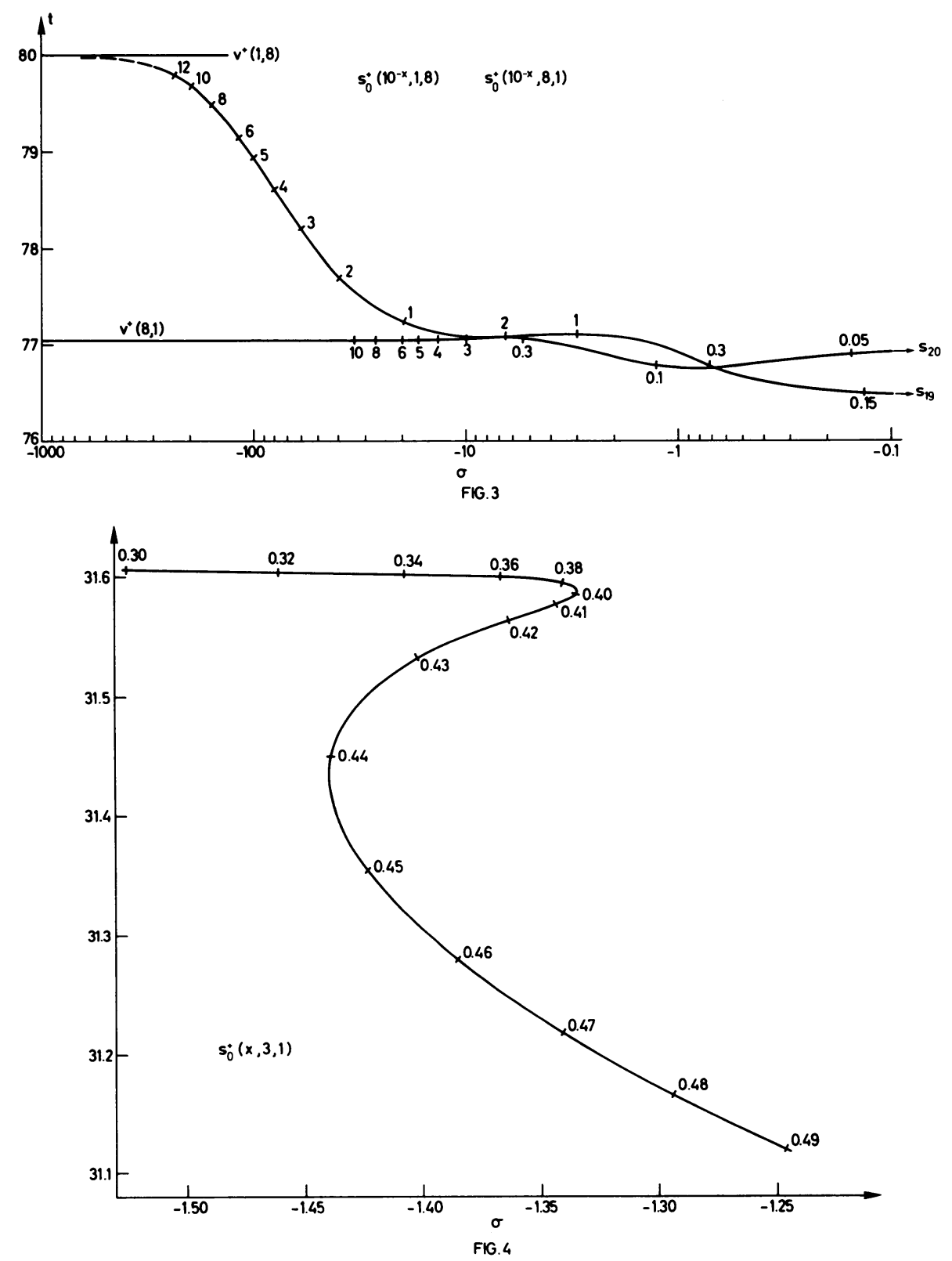

Since $|\Gamma(1-s)|$, in the neighbourhood of the first nontrivial zero $s_{1}=1 / 2+14.134725 i$ is of the order $10^{-10}$ (and decreases strongly for an increasing imaginary part of $s$ ), $\Psi(x, s)$ is also small near $s_{1}$ for $x$ less than, say, $1-10^{-8}$. This term has therefore essentially no effect on the zero of $F(x, s)$ in the neighbourhood of $s_{1}$. When $x$ is even closer to one, $\log (-\log x)$ decreases to $-\infty$. Near $s_{1}$, the function $\Psi(x, s)$ increases exponentially from a very small value; and the phase rotates with an angle proportional to $\log (-\log x)$. The zero [i.e., the point where this term cancels $\zeta(s)$, which has a simple zero at $s_{1}$ ] therefore starts to rotate in an exponential spiral around $s_{1}$. 


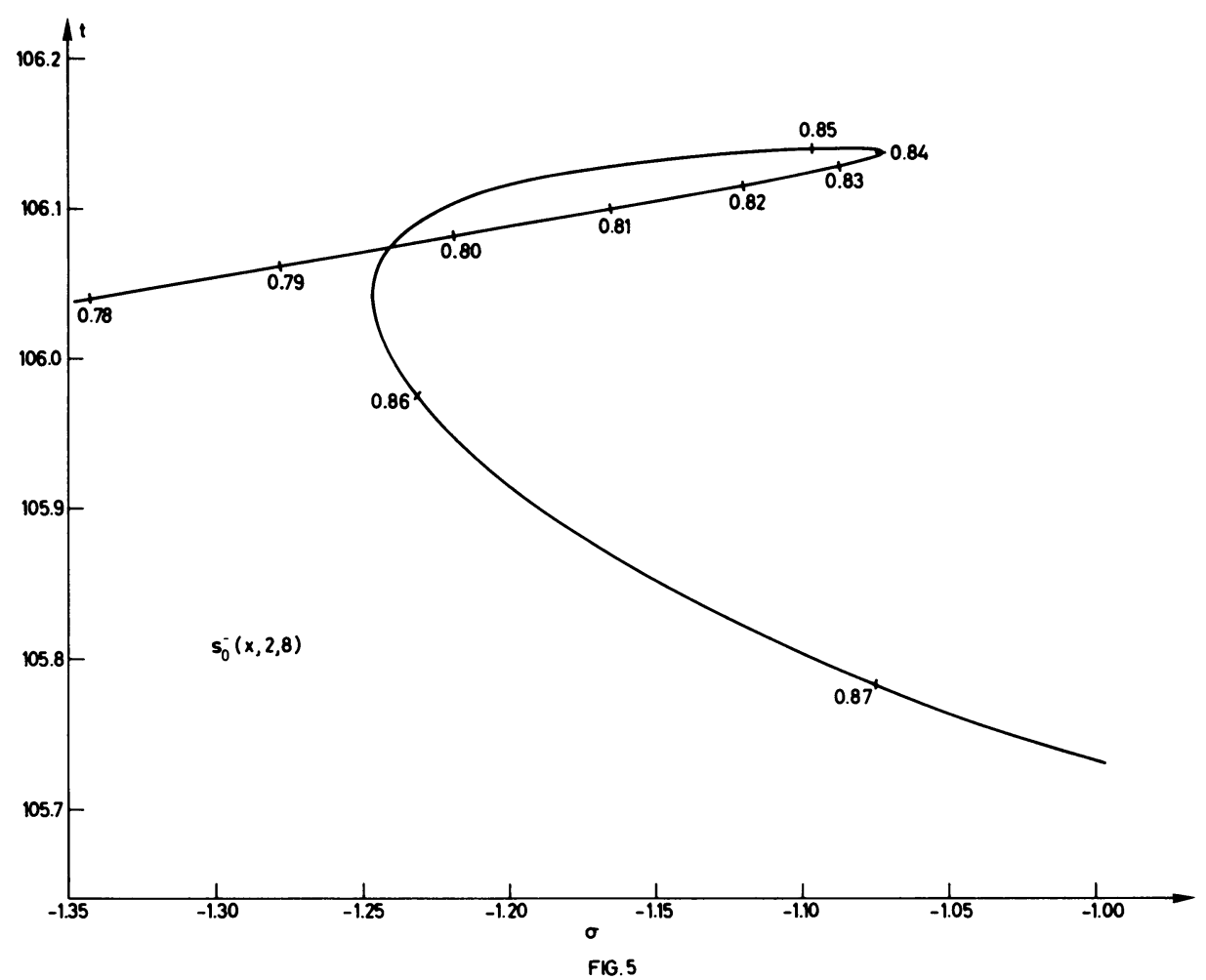

To see what happens when $x$ approaches one even more closely, we divide (26) by $\Gamma(1-s)$. Again ignoring the sum in (26), we study the points where $-\zeta(s) / \Gamma(1-s)$ is equal to $\exp [(s-1) \log (-\log x)]$. For $x \rightarrow 1$, $\exp [(s-1) \log (-\log x)] \mid$ tends to infinity at every point $s$ for which $\operatorname{Re} s<1$ and tends to zero for those having $\operatorname{Re} s>1$. The curve along which $-\zeta(s) / \Gamma(1-s)$ (which is a regular function for $s=1$ ) and $\exp [(s-1) \log (-\log x)]$ agree in magnitude thus approaches the line Re $s=1$. [Although $-\zeta(s) / \Gamma(1-s)$ is zero for $s=2,3,4, \cdots, F(x, s)$ has no zeros in the neighbourhood of these points. The poles of $\Gamma(1-s)$ are cancelled by the poles of $\zeta(s-n)$ in the sum on the right-hand side of (26).] Along this curve, tending to $\operatorname{Re} s=1$, the phase of $-\zeta(s) / \Gamma(1-s)$ varies slowly, relative to the phase of $\exp [(s-1) \log (-\log x)]$, and $F(x, s)$ has zeros when the phase of $-\zeta(s) / \Gamma(1-s)$ agrees with the phase of $\exp [(s-1) \log (-\log x)]$. This occurs at almost equidistant points [asymptotically spaced at distance $2 \pi /|\log (-\log x)|]$ all tending to $\operatorname{Im} s=0$, and thus tending to $s=1$ when $x \rightarrow 1$.

The explanations given in the above paragraph for the behaviour near $s=1$ are also valid for those trajectories which tend directly to $s=1$.

Near $s=1$, the zero trajectories $s_{0}^{+}(x, 0, N)$ were computed in the following way. The method described in Section 3 was used up to $x=0.98$. For $0.98<x \leqslant 1-$ $10^{-10}$, the functional equation

$$
F(x, s)=2^{1-s} F\left(x^{2}, s\right)-F(-x, s)
$$



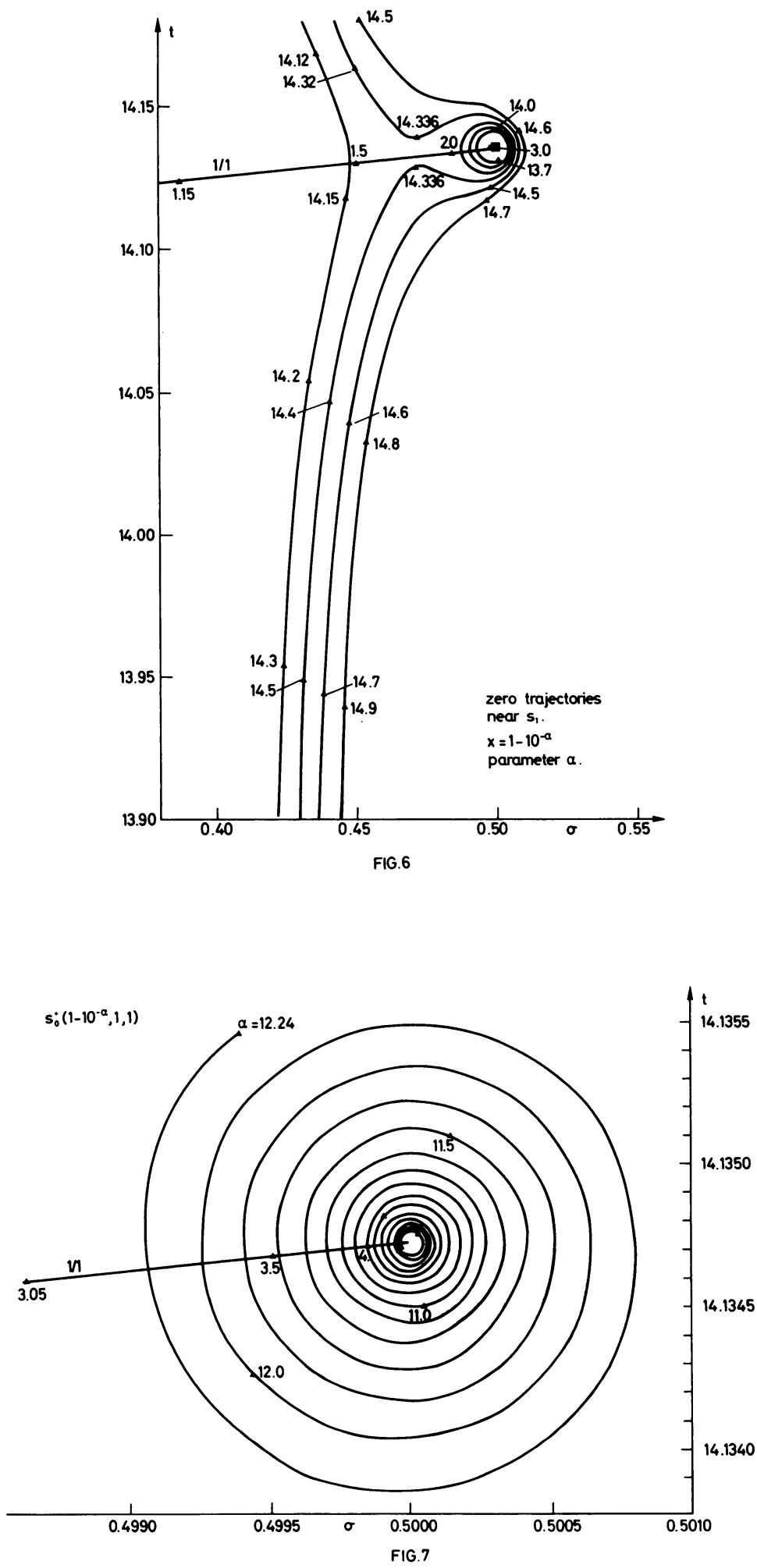
was applied. The series $F(-x, s)$ can be summed by means of the Euler transformation [17]. Convergence is then achieved essentially from the fact that $1 / k^{s}$ behaves smoothly as a function of $k$ and not so much from the additional factors $x^{k}$. The problem of calculating $F(x, s)$ for $x$ close to one is thus reduced to the calculation of $F\left(x^{2}, s\right)$, i.e., the same problem with a smaller argument $x$. This recursion was used until the value of $x$ by successive squarings had decreased below 0.5 , when a direct summation was done. For example, in the case of $x=1-10^{-10}$, this required 33 steps in the recursion.

For the first trajectory $N=P=1$, we investigated numerically the behaviour near the point $s_{1}$, setting $x=1-10^{-\alpha}$. Up to $\alpha=10$, the recursion method (28) was used, and, for $\alpha>10$, formula (26) was used, ignoring the sum term. Figure 6 shows how the trajectory $s_{0}^{+}(x, 1,1)$ initially approaches $s_{1}$ and later leaves it in the way described above. The figure also shows three other zero trajectories which pass close to $s_{1}$ and join $s_{0}^{+}(x, 1,1)$ on its way to $s=1$. The small rectangle at the centre of the spiral in Fig. 6 encloses the region which is shown on an enlarged scale in Fig. 7.

For $x \rightarrow-1$, in view of the convergence of the series (1) for $\operatorname{Re} s=\sigma>0$ and $x=-1$, we believe that the trajectories $s_{0}^{-}(x, P, N)$ actually converge to $s_{m}$ or $s_{m}^{\prime}$.

6. Trajectories Corresponding to the Trivial Zeros of $\zeta(s)$. The function $\zeta(s)$ has, apart from the zeros in the strip $0<\sigma<1$, so-called trivial zeros at the points

$$
s_{m}^{\prime \prime}=-2 m \quad(m=1,2,3, \cdots) .
$$

These zeros are approached by trajectories $s_{0}^{-}(x, 0, N)=u^{-}(x, 0, N)$ lying on the real axis. There are no trajectories $s_{0}^{+}(x, P, N)$ going to the trivial zeros.

7. The Number of Asymptotes. It is well known [18] that the number $N(T)$ of zeros $s_{m}$ of $\zeta(s)$, for which $t$ lies in the interval of $0<t \leqslant T$, can be expressed for $T \rightarrow \infty$ by

$$
N(T)=\frac{T}{2 \pi} \log T-\frac{1+\log 2 \pi}{2 \pi} T+R(T)
$$

where $R(T)=O(\log T)$. Since it was found that the first 33 zeros $s_{m}$ could be assigned to corresponding asymptotes $v^{+}(P, N)$ and $v^{-}(P, N)$, it would be interesting to know the number of asymptotes in the interval $0<t \leqslant T$ for $T \rightarrow \infty$. As was mentioned in Section 4 , the imaginary part of a zero $s_{m}$ which is approached by a trajectory $s_{0}(x, P, N)$ may differ considerably from the $t$ value of the corresponding asymptote $v(P, N)$; so one cannot necessarily expect a formula identical to that for $N(T)$.

It follows from (17) that the problem of finding the number $A^{+}(T)$ of asymptotes $v^{+}(N, P)$ in $0<t \leqslant T$ is equivalent to counting the number of lattice points in the region $(P \geqslant 1, N \geqslant 1)$ which lie below or on the curve

$$
N_{T}(P)=\left\{\exp \frac{(2 P+1) \pi}{T}-1\right\}^{-1}
$$


or, equivalently, which lie on the left-hand side of, or on, the curve

$$
P_{T}(N)=(T / 2 \pi) \log (1+1 / N)-1 / 2 .
$$

Therefore, following an idea of Hardy and Wright [19], we can write

$$
A^{+}(T)=\sum_{P=1}^{P *}\left[N_{T}(P)\right]+\sum_{N=1}^{N^{*}}\left[P_{T}(N)\right]-P^{*} N^{*}
$$

where $P^{*}=\left[T^{1 / 2}\right]$ and $N^{*}=\left[N_{T}\left(P^{*}\right)\right]$, and $[\xi]$ is the integer part of $\xi$. If we are satisfied with an expression for $A^{+}(T)$ which is, for $T \rightarrow \infty$, correct up to $O\left(T^{1 / 2}\right)$, we can proceed in the following way. Because of the fact that

$$
P^{*}=T^{1 / 2}+O(1) \text { and } N^{*}=T^{1 / 2} / 2 \pi+O(1),
$$

we get

$$
A^{+}(T)=\sum_{P=1}^{P^{*}}\left\{\exp \frac{(2 P+1) \pi}{T}-1\right\}^{-1}+\frac{T}{2 \pi} \sum_{N=1}^{N^{*}} \log \left(1+\frac{1}{N}\right)-\frac{T}{2 \pi}+O\left(T^{1 / 2}\right)
$$

$$
=\frac{T}{\pi} \sum_{P=1}^{P *} \frac{1}{2 P+1}+\frac{T}{2 \pi} \log \left(N^{*}+1\right)-\frac{T}{2 \pi}+O\left(T^{1 / 2}\right) .
$$

Using the well-known formula [20],

$$
\sum_{k=1}^{n} \frac{1}{2 k+1}=-1+\frac{1}{2} \gamma+\frac{1}{2} \log n+\log 2+O\left(n^{-2}\right) \quad(n \rightarrow \infty),
$$

where $\gamma=0.577216$ is Euler's constant, and the relations (34), we finally obtain

$$
A^{+}(T)=\frac{T}{2 \pi} \log T-\frac{3+\log 2 \pi-2 \log 2-\gamma}{2 \pi} T+O\left(T^{1 / 2}\right) \quad(T \rightarrow \infty) .
$$

Similarly, one finds for the number $A^{-}(T)$ of asymptotes $v^{-}(P, N)(P \geqslant 1, N \geqslant 2)$, in the interval $0<t \leqslant T$, the expression

$$
A^{-}(T)=\frac{T}{2 \pi} \log T-\frac{1+\log 2 \pi+\log 2-\gamma}{2 \pi} T+O\left(T^{1 / 2}\right) \quad(T \rightarrow \infty) .
$$

Comparing (37) and (38) with the expression (30) for $N(T)$, we see that, for large $T$, there are in both cases more zeros $s_{m}$ than asymptotes in the interval $0<t \leqslant T$, the excess being proportional to $T$, namely

$$
N(T)-A^{ \pm}(T)=\alpha^{ \pm} T+O\left(T^{1 / 2}\right),
$$

where

$$
\alpha^{+}=\frac{2-2 \log 2-\gamma}{2 \pi}=0.00580756, \quad \alpha^{-}=\frac{\log 2-\gamma}{2 \pi}=0.01845107 .
$$

In order to get an idea of the magnitude of the $O\left(T^{1 / 2}\right)$ term, we carried out a computer calculation of the numbers $A^{+}(T)$ and $A^{-}(T)$ for $T=10^{j}, j=1(1) 12$. It was found that the computed values of $\alpha^{+}$and $\alpha^{-}$converge for increasing $j$, and that for $T=10^{12}$ 
they agree, to eight decimal places, with the values (40). It should be noted that $P=$ 0 is not counted for $A^{+}(T)$, and that $N=1$ is not counted for $A^{-}(T)$, for the reasons described in Section 4. The inclusion of these asymptotes would lead to values

$$
\alpha^{+}=-\frac{2 \log 2+\gamma}{2 \pi}=-0.3125023, \quad \alpha^{-}=-\frac{\gamma}{2 \pi}=-0.0918667,
$$

which have the opposite sign and are larger in magnitude than those obtained in (40).

Acknowledgements. We wish to thank G. A. Erskine and B. Schorr for helpful and encouraging discussions, and Miss E. Vial for the careful drawing of the figures.

\section{CERN}

1211 Geneva 23

Switzerland

1. W. MAGNUS, F. OBERHETTINGER \& R. P. SONI, Formulas and Theorems for the Special Functions of Mathematical Physics, Die Grundlehren der math. Wissenschaften, Bd. 52, Springer-Verlag, New York, 1966. MR 38 \#1291.

2. A. ERDÉLYI, W. MAGNUS, F. OBERHETTINGER \& F. G. TRICOMI, Higher Trancendental Functions, vol. I, McGraw-Hill, New York, 1953. MR 15, 419.

3. R. B. DINGLE, Asymptotic Expansions: Their Derivation and Interpretation, Academic Press, London and New York, 1973.

4. L. LEWIN, Dilogarithms and Associated Functions, Macdonald, London, 1958. MR 21 $\# 4264$.

5. N. NIELSEN, "Der Eulersche Dilogarithmus und seine Verallgemeinerungen," Nova Acta Leopoldina Halle, v. 90, 1909, pp. 123-211.

6. G. WECHSUNG, "Lineare Funktionalgleichungen von Polylogarithmen," Wiss. Z. FriedrichSchiller-Univ. Jena/Thüringen, v. 14, 1965, pp. 401-408. MR 37 \#6638.

7. W. MAIER \& H. KIESEWETTER, Funktionalgleichungen mit analytischen Lösungen, VEB Deutscher Verlag der Wissenschaften, Berlin, 1971.

8. D. MAISON \& A. PETERMANN, "Subtracted generalized polylogarithms and the SINAC program," Comput. Phys. Comm., v. 7, 1974, pp. 121-134.

9. G. WECHSUNG, “Logarithmische Integrale," Publ. Math. Debrecen, v. 14, 1967, pp. 255-271. MR 36 \#6655.

10. K. S. KÖLBIG, J. A. MIGNACO \& E. REMIDDI, “On Nielsen's generalized polylogarithms and their numerical calculation," Nordisk Tidskr. Informationsbehandling (BIT), v. 10, 1970, pp. 38-74. MR 44 \#2967.

11. D. JACOBS \& F. LAMBERT, “On the numerical calculation of polylogarithms," Nordisk Tidskr. Informationsbehandling (BIT), v. 12, 1972, pp. 581-585.

12. R. H. BARLOW, "Convergent continued fraction approximants to generalized polylogarithms," Nordisk Tidskr. Informationsbehandling (BIT), v. 14, 1974, pp. 112-116.

13. W. F. PICKARD, “On polylogarithms," Publ. Math. Debrecen, v. 15, 1968, pp. 33-43. MR 38 \#6133.

14. A. KOPPÁNYI, NEWTON-Solution of Simultaneous Non-Linear Equations, CERN 7600 Program Library C400, 1972 (unpublished).

15. C. B. HASELGROVE \& J. C. P. MILLER, Tables of the Riemann Zeta Function, Roy. Soc. Math. Tables, vol. 6, Cambridge Univ. Press, New York, $1960 . \quad$ MR 22 \#8679.

16. G. A. ERSKINE \& K. S. KÖLBIG, CGAUSS-Complex Integration Along a Line Segment, CERN 7600 Program Library D113, 1970 (unpublished).

17. D. R. HARTREE, Numerical Analysis, 2nd ed., Oxford Univ. Press, New York, 1958. MR 20 \#6768.

18. E. C. TITCHMARSH, The Theory of the Riemann Zeta-Function, Clarendon Press, Oxford, 1967.

19. G. H. HARDY \& E. M. WRIGHT, An Introduction to the Theory of Numbers, 3rd ed., Clarendon Press, Oxford, 1954. MR 16, 673.

20. I. M. RYSHIK \& I. S. GRADSTEIN, Tafeln-Tables, VEB Deutscher Verlag der Wissenschaften, Berlin, 1957. 